\title{
Performance of the Phoenix bacterial identification system compared with disc diffusion methods for identifying extended-spectrum $\beta$-lactamase, AmpC and KPC producers
}

Correspondence

Mark A. Fisher

mark.fisher@path.utah.edu

Received 28 August 2008

Accepted 5 February 2009

\author{
Mark A. Fisher, ${ }^{1,2}$ Paul D. Stamper, ${ }^{3}$ Kristine M. Hujer, ${ }^{4}$ Zachary Love, ${ }^{5}$ \\ Ann Croft, ${ }^{2}$ Samuel Cohen, ${ }^{2}$ Robert A. Bonomo, ${ }^{4,5}$ Karen C. Carroll ${ }^{3}$ \\ and Cathy A. Petti ${ }^{1,2}$ \\ ${ }^{1}$ University of Utah, School of Medicine, Salt Lake City, UT, USA \\ ${ }^{2}$ ARUP Laboratories, Salt Lake City, UT, USA \\ ${ }^{3}$ The Johns Hopkins Hospital, Baltimore, MD, USA \\ ${ }^{4}$ Louis Stokes VA Medical Center, Cleveland, Ohio, USA \\ ${ }^{5}$ Case Western Reserve University, School of Medicine, Cleveland, OH, USA
}

\begin{abstract}
Phenotypic identification of AmpC, KPC and extended-spectrum $\beta$-lactamases (ESBLs) among members of the Enterobacteriaceae remains challenging. This study compared the Phoenix Automated Microbiology System (BD Diagnostics) with the Clinical and Laboratory Standards Institute confirmatory method to identify ESBL production among 200 Escherichia coli and Klebsiella pneumoniae clinical isolates. The Phoenix system misclassified nearly half of the isolates as ESBL-positive, requiring manual testing for confirmation. Inclusion of aztreonam \pm clavulanic acid $(C A)$ and cefpodoxime $\pm C A$ in the testing algorithm increased the ESBL detection rate by $6 \%$. Boronic acid-based screening identified 24 isolates as $\mathrm{AmpC}^{+}$, but in a subset of genotypically characterized isolates, appeared to have a high false-positivity rate. $\mathrm{PCR}$ screening revealed eight $\mathrm{KPC}^{+}$isolates, all of which tested as $\mathrm{ESBL}^{+}$or $\mathrm{ESBL}^{+} \mathrm{AmpC}^{+}$ by phenotypic methods, but half were reported as carbapenem-susceptible by the Phoenix system. Overall, these results indicate that laboratories should use the Phoenix ESBL results only as an initial screen followed by confirmation with an alternative method.
\end{abstract}

\section{INTRODUCTION}

Extended-spectrum $\beta$-lactamases (ESBLs), AmpC and KPC $\beta$-lactamases are important mechanisms of $\beta$-lactam resistance among members of the Enterobacteriaceae. Accurate detection of $\beta$-lactamase producers is critical for therapeutic and infection control decision-making. The Clinical and Laboratory Standards Institute (CLSI, 2008) has standardized methods for ESBL detection, but many laboratories rely on automated systems for antimicrobial susceptibility testing. Unfortunately, some automated systems have difficulty detecting ESBLs, AmpCs and KPCs (Tenover et al., 2006; Wiegand et al., 2007). With the recent implementation of the Phoenix Automated Microbiology System (BD Diagnostics), we observed a higher frequency of $\mathrm{ESBL}^{+}$calls among Escherichia coli and Klebsiella pneumoniae isolates compared with the CLSI disc diffusion (DD) method.

Abbreviations: $\mathrm{BA}$, boronic acid; $\mathrm{CA}$, clavulanic acid; $\mathrm{DD}$, disc diffusion; ESBL, extended-spectrum $\beta$-lactamase.
Incorporation of manual methods in an ESBL-testing algorithm for laboratories using automated instruments has not been well delineated. To determine the optimal algorithm, we (i) compared the CLSI and Phoenix methods for identifying $\mathrm{ESBL}^{+}$isolates of E. coli and K. pneumoniae, (ii) assessed the value of an extended phenotypic method [cefpodoxime and aztreonam \pm clavulanic acid (CA)] for enhancing ESBL detection, (iii) evaluated boronic acid (BA) with cefotetan or ceftazidime \pm CA to identify AmpC producers that could mimic or mask ESBL production, and (iv) assessed these phenotypic tests with KPC-positive clinical isolates.

\section{METHODS}

Bacterial isolates. Two hundred isolates of E. coli $(n=119)$ and $K$. pneumoniae $(n=81)$ were collected at two sites representing urban (The Johns Hopkins Hospital, Baltimore, MD, USA) and geographically diverse (ARUP Laboratories, Salt Lake City, UT, USA) populations. Identification and susceptibility testing were performed 
on a Phoenix system (instrument version 5.15A, software version 5.10A/V4.31A), using a standard protocol, at both sites. One hundred consecutive isolates flagged as $\mathrm{ESBL}^{+}$by the Phoenix system and 93 randomly selected $\mathrm{ESBL}^{-}$isolates were collected. Seven isolates resistant to a third-generation cephalosporin or aztreonam but called $\mathrm{ESBL}^{-}$by the Phoenix system were selected for inclusion.

Phenotypic identification of ESBL and AmpC producers. All Phoenix ESBL ${ }^{+}$and ESBL ${ }^{-}$isolates were tested by DD for ESBL (CLSI, 2008) and AmpC production as described previously (Coudron, 2005). Discs containing antibiotics [30 $\mu \mathrm{g}$ ceftazidime, $30 \mu \mathrm{g}$ cefotaxime, $10 \mu \mathrm{g}$ cefpodoxime, $30 \mu \mathrm{g}$ aztreonam (BD Diagnostics) or $30 \mu \mathrm{g}$ cefotetan (Remel)] with or without $\beta$-lactamase inhibitors [10 $\mu \mathrm{g} \mathrm{CA}$ (GlaxoSmithKline) or $400 \mu \mathrm{g}$ BA (Sigma)] were placed on MuellerHinton agar streaked with the test organism and incubated at $35{ }^{\circ} \mathrm{C}$ for $16-18 \mathrm{~h}$ in ambient air. In total, $12 \mathrm{drug} /$ inhibitor combinations (ceftazidime $\pm \mathrm{CA}$ and cefotaxime $\pm \mathrm{CA}$ for the CLSI ESBL test, cefpodoxime $\pm \mathrm{CA}$ and aztreonam $\pm \mathrm{CA}$ for the $\mathrm{ESBL}$ test, cefotetan $\pm \mathrm{BA}$ for $\mathrm{AmpC}$ detection, and ceftazidime $+\mathrm{BA} \pm \mathrm{CA}$ for AmpC-masked ESBL detection) were tested for each isolate. Results were considered positive (indicative of $\beta$-lactamase) for any drug showing $\mathrm{a} \geqslant 5 \mathrm{~mm}$ increase in zone of inhibition with an inhibitor.

Molecular characterization of resistance mechanisms. A random subset of 24 isolates determined as $\mathrm{ESBL}^{+}$by the Phoenix system were examined for ESBL, AmpC and KPC $\beta$-lactamases by IEF, PCR and sequencing to confirm the phenotypic findings. Seven isolates determined as $\mathrm{ESBL}^{-}$by the Phoenix assay but $\mathrm{ESBL}^{+}$and/or $\mathrm{AmpC}^{+}$by DD were also characterized by molecular methods.

IEF was performed by loading $10 \mu \mathrm{l}$ of a crude enzyme extract onto pre-cast gels (Ampholine PAGplate, pH 3.5-9.5; GE Healthcare). Purified TEM and SHV enzymes and isolates with previously characterized $\beta$-lactamases were used as controls (Paterson et al., 2001, 2003). PCR amplification was performed on $10 \mu \mathrm{l}$ aliquots of diluted lysates from overnight cultures using primers specific for $b l a_{\mathrm{TEM}}, b l a_{\mathrm{SHV}}, b l a_{\mathrm{CTX}-\mathrm{M}}, b l a_{\mathrm{PER}}, b l a_{\mathrm{ACT}}, b l a_{\mathrm{MIR}}, b l a_{\mathrm{CMY}}, b l a_{\mathrm{BIL}}, b l a_{\mathrm{LAT}}$ and $b l a_{\mathrm{DHA}}$ genes as described previously (Paterson et al., 2003). DNA sequencing was performed (ALFexpress, Thermo Sequenase; GE Healthcare) to identify TEM and SHV ESBLs by comparison with previously characterized enzymes (http://www.lahey.org/Studies) and to subtype $\mathrm{KPC}^{+}$isolates. This group of 31 well-characterized isolates served as a reference set by which to compare phenotypic methods. In addition, all 200 isolates were screened by PCR for the $b l a_{\mathrm{KPC}}$ gene as described previously (Smith Moland et al., 2003).

\section{RESULTS AND DISCUSSION}

\section{ESBL detection}

Of 100 isolates determined as $\mathrm{ESBL}^{+}$by the Phoenix system, only 51 were verified by the CLSI confirmatory method (Table 1). Even the extended testing algorithm, including cefpodoxime $\pm \mathrm{CA}$ and aztreonam $\pm \mathrm{CA}$, confirmed only $54 \%$ of the isolates determined as $\mathrm{ESBL}^{+}$by the Phoenix system. Of 100 isolates determined as ESBL by the Phoenix test (60 E. coli and $40 \mathrm{~K}$. pneumoniae), $96 \%$ were confirmed by the DD methods (Table 1).

Molecular analyses on a subset of isolates confirmed the DD results for identification of ESBLs. Twenty-seven of 31 isolates were concordant between $\mathrm{DD}$ and molecular testing, whereas only nine (29\%) were concordant between the molecular and Phoenix ESBL results (Table 2). Two
Table 1. Summary of phenotypic ESBL and AmpC classification of 200 clinical isolates categorized as $\mathrm{ESBL}^{+}(n=100)$ or $\mathrm{ESBL}^{-}(n=100)$ by the Phoenix system

Results in parentheses show the number of E. coli and K. pneumoniae, respectively.

\begin{tabular}{|lcc|}
\hline Phenotype $^{*}$ & ESBL $^{+}$by Phoenix & ESBL $^{-}$by Phoenix \\
\hline $\mathrm{ESBL}^{+}$ & $46(19,27) \dagger$ & $2(1,1)$ \\
$\mathrm{ESBL}^{+} \mathrm{AmpC}^{+}$ & $8(1,7) \ddagger$ & $2(0,2) \S$ \\
$\mathrm{AmpC}^{+}$ & $11(11,0)$ & $3(2,1)$ \\
$\mathrm{ESBL}^{-} \mathrm{AmpC}^{-}$ & $35(28,7)$ & $93(57,36)$ \\
Total & $100(59,41)$ & $100(60,40)$ \\
\hline
\end{tabular}

${ }^{\star}$ Determined by DD testing as described in the text.

$\dagger$ One E. coli and one K. pneumoniae were determined as $\mathrm{ESBL}^{+}$only by the extended method.

‡One K. pneumoniae was determined as $\mathrm{ESBL}^{+}$only by the extended method.

§oth isolates were determined as $\mathrm{ESBL}^{+}$only by the extended method.

isolates (101 and 118) were determined as $\mathrm{ESBL}^{+}$by DD and Phoenix testing but were $\mathrm{ESBL}^{-} \mathrm{KPC}^{+}$by PCR. This was not unexpected as KPC enzymes are CA-sensitive and can behave phenotypically like ESBLs (Jacoby \& MunozPrice, 2005). Of seven isolates determined as $\mathrm{ESBL}^{-}$by the Phoenix system but $\mathrm{ESBL}^{+}$and/or $\mathrm{AmpC}^{+}$by DD testing, the molecular results agreed with the DD results in every case. Overall, the molecular data supported the DD results, indicating that the Phoenix system was overcalling ESBLs.

Our data confirm the high sensitivity (93\%) but questionable specificity $(68 \%)$ reported for Phoenix ESBL detection (Thomson et al., 2007; Wiegand et al., 2007). Because isolates producing false-positive and falsenegative results in the CLSI test have been described (Thomson et al., 2007), it is not considered the gold standard for ESBL detection. However, it is a robust assay that is the mainstay of ESBL testing in many laboratories (Steward et al., 2001; Tenover et al., 2003). Our molecular data support the CLSI results and suggest that it is unlikely that the CLSI-confounding isolates were present in sufficient numbers to explain the 49 discrepant ESBL calls. The extended DD method increased the detection of ESBLs by $6 \%$ compared with the traditional CLSI method, which may warrant adoption in areas with high ESBL prevalence. Based on these results, we recommend the Phoenix ESBL test be used only as an initial screen for ESBLs, followed by confirmation with an alternative test such as the CLSI method or a molecular assay.

\section{AmpC detection}

DD testing with BA as an inhibitor identified 24 putative $\mathrm{AmpC}^{+}$isolates, including $10 \mathrm{AmpC}^{+} \mathrm{ESBL}^{+}$isolates (Table 1). Only one isolate, 101, showed an AmpC-masked 
Table 2. Genotypic and phenotypic $\beta$-lactamase results from 31 selected isolates

\begin{tabular}{|c|c|c|c|c|c|c|c|}
\hline \multirow[t]{2}{*}{ Isolate no. } & \multirow[t]{2}{*}{ Species } & \multicolumn{3}{|c|}{ Genotype $^{*}$} & \multirow[t]{2}{*}{ Phoenix ESBL $\dagger$} & \multicolumn{2}{|c|}{$\mathrm{DD} \ddagger$} \\
\hline & & ESBL & AmpC & KPC & & ESBL & AmpC \\
\hline A13 & E. coli & - & - & - & + & - & - \\
\hline A15 & E. coli & - & + & - & + & - & + \\
\hline D2 & K. pneumoniae & - & - & - & + & + & + \\
\hline $\mathrm{F} 4$ & E. coli & - & + & - & - & - & + \\
\hline F6 & E. coli & CTX-M & - & - & - & + & - \\
\hline F9 & K. pneumoniae & SHV-28, CTX-M & - & - & + & + & - \\
\hline G7 & K. pneumoniae & - & - & - & + & - & - \\
\hline $\mathrm{H} 2$ & K. pneumoniae & SHV-12 & + & - & - & + & + \\
\hline 109 & E. coli & - & - & - & + & - & - \\
\hline 113 & E. coli & - & - & - & + & - & + \\
\hline 116 & E. coli & - & - & - & + & - & - \\
\hline 118 & K. pneumoniae & - & - & + & + & + & + \\
\hline 119 & K. pneumoniae & SHV-12 & - & - & + & + & - \\
\hline 124 & K. pneumoniae & - & - & - & + & - & - \\
\hline 126 & E. coli & CTX-M & - & - & + & + & + \\
\hline 140 & E. coli & CTX-M & - & - & + & + & - \\
\hline 141 & E. coli & - & - & - & + & - & - \\
\hline 144 & E. coli & - & - & - & + & - & + \\
\hline 146 & E. coli & - & - & - & + & - & - \\
\hline
\end{tabular}

${ }^{\star}$ TEM, SHV and KPC PCR products were sequenced to identify bla subtypes. Both $\mathrm{KPC}^{+}$isolates were type 3 based on sequence analysis. $\dagger$ ESBL results determined using the Phoenix system.

$¥$ Phenotypes were determined by DD testing as described in the text.

$\S$ This isolate was determined as ESBL only using ceftazidime $+\mathrm{BA} \pm \mathrm{CA}$.

ESBL phenotype (increased susceptibility to ceftazidime only with both BA and CA). Molecular analyses revealed that Phoenix and DD testing misidentified this KPC-3 producer as $\mathrm{ESBL}^{+}$and $\mathrm{ESBL}^{+} \mathrm{AmpC}^{+}$, respectively (Table 2). This is not surprising as KPCs can be sensitive to both CA and BA inhibition (Jacoby \& Munoz-Price, 2005; Livermore et al., 2007; Pasteran et al., 2008). Of the 10 isolates determined as $\mathrm{AmpC}^{+} \mathrm{ESBL}^{+}$by $\mathrm{BA}$ and CA inhibition, the CLSI and Phoenix methods classified 70 and $80 \%$ as $\mathrm{ESBL}^{+}$, respectively. Of the $14 \mathrm{AmpC}^{+} \mathrm{ESBL}^{-}$ isolates, only $3(21 \%)$ were correctly determined as $\mathrm{ESBL}^{-}$ by the Phoenix system (Table 1).

Molecular testing did not correlate well with DD results for AmpC detection. Only six of the 13 phenotypically $\mathrm{AmpC}^{+}$isolates were found to be $\mathrm{AmpC}^{+}$by molecular testing (Table 2), suggesting that the BA method overcalled
AmpC producers among these isolates. A limitation of using BA to identify AmpC producers is that it does not inhibit AmpC hydrolysis of all cephalosporins (Jacoby et al., 2006; Yagi et al., 2005). However, cefotetan \pm BA, as used here, previously detected nearly $90 \%$ of AmpC enzymes in control strains (Coudron, 2005). The AmpC primers we used were specific for only a subset of AmpC genes (Paterson et al., 2003), which may explain discrepancies between phenotypic and molecular data for our 31 reference isolates. A broader set of primers, such as those used by Pérez-Pérez \& Hanson (2002), may confirm more $\mathrm{AmpC}^{+}$isolates. Given the current data, including confirmation that KPC producers can be sensitive to BA, like AmpCs (isolate 101, Table 2), we cannot recommend cefotetan $\pm \mathrm{BA}$ as a stand-alone test for AmpC detection. 
Table 3. Cephalosporin and carbapenem susceptibility and MIC data from $\mathrm{KPC}^{+}$isolates

All isolates were K. pneumoniae and categorized as $\mathrm{ESBL}^{+}$using the Phoenix system.

\begin{tabular}{|c|c|c|c|c|c|c|c|c|c|c|}
\hline \multirow[t]{2}{*}{ Isolate no. } & \multicolumn{5}{|c|}{ DD interpretation ${ }^{\star}$} & \multicolumn{4}{|c|}{$\operatorname{MIC}\left(\mu \mathrm{g} \mathrm{ml}^{-1}\right) \dagger$} & \multirow[t]{2}{*}{ Phenotype $\neq$} \\
\hline & Atm & $\mathrm{Ctt}$ & Cpd & Ctx & $\mathrm{Caz}$ & Cro & Fep & Ipm & Mem & \\
\hline 107 & $\mathrm{R}$ & S & $\mathrm{R}$ & S & I & $\underline{32}$ & 2 & $<=1$ & $<=1$ & ESBL \\
\hline 117 & I & S & $\mathrm{R}$ & S & I & $\overline{32}$ & 2 & 8 & $<=1$ & ESBL \\
\hline 118 & $\mathrm{R}$ & $\mathrm{R}$ & $\mathrm{R}$ & $\mathrm{R}$ & $\mathrm{R}$ & $>32$ & 4 & $<=1$ & $<=1$ & $\mathrm{ESBL}+\mathrm{AmpC}$ \\
\hline 167 & $\mathrm{R}$ & $\mathrm{R}$ & $\mathrm{R}$ & $\mathrm{R}$ & $\mathrm{R}$ & $>32$ & $>16$ & $>8$ & $>8$ & $\mathrm{ESBL}+\mathrm{AmpC}$ \\
\hline 171 & $\mathrm{R}$ & S & $\mathrm{R}$ & I & $\mathrm{R}$ & $>32$ & 8 & $<=1$ & $<=1$ & ESBL \\
\hline
\end{tabular}

${ }^{\star}$ Interpretations based on zone size using CLSI criteria. S, Susceptible; I, intermediate susceptibility; R, resistant. Atm, aztreonam; Ctt, cefotetan; Cpd, cefpodoxime; Ctx, cefotaxime; Caz, ceftazidime.

$\dagger$ MICs were determined by the Phoenix system. Bold indicates resistance and underlining indicates intermediate susceptibility according to CLSI criteria. Cro, Ceftriaxone; Fep, cefepime; Ipm, imipenem; Mem, meropenem.

$\$$ Phenotypes were determined by DD testing as described in the text.

\section{KPC detection}

A PCR screen of our 200 clinical isolates revealed eight carrying the $b l a_{\mathrm{KPC}}$ gene. Although both phenotypic methods indicated $\beta$-lactamase production (ESBL and/or AmpC) in these isolates, the Phoenix system unfortunately reported half as meropenem- and imipenem-susceptible (Table 3). Many automated instruments fail to detect carbapenemases, which is a serious concern in the laboratory (Tenover et al., 2006). Ertapenem may be a more sensitive indicator of KPC production, but was not included on the Phoenix panels used in this or a previous study (Anderson et al., 2007). Overall, these observations highlight the significant presence of $\mathrm{KPC}^{+}$isolates in the USA and the need for more reliable detection methods.

\section{Conclusions}

From 100 isolates, the Phoenix system made four falsenegative and 46 false-positive ESBL calls relative to DD testing. Of the 20 discrepants tested by molecular methods, DD results were confirmed for all four false-negatives and $94 \%$ of false-positives. In addition, half of the $\mathrm{KPC}^{+}$ isolates were reported as carbapenem-susceptible by the Phoenix system. These errors can adversely impact on the healthcare system in a number of ways, including inappropriate therapy and inaccurate antimicrobial surveillance data.

These results show that the Phoenix system overcalled ESBLs relative to the CLSI confirmatory and molecular testing. Neither the Phoenix system nor DD testing appear to be robust enough for routine detection of $\mathrm{AmpC}^{+}$or $\mathrm{KPC}^{+}$isolates, stressing the need for reliable methods to detect these important resistance mechanisms in the clinical laboratory.

\section{ACKNOWLEDGEMENTS}

We thank Barbara Schaecher for assistance in collecting and organizing isolates and Dustin Williams for assistance with the KPC screens. This work was supported in part by the National Institutes of Health (RO1-AI63517) and the Veterans Affairs Merit Review Program (K. M. H., Z. L., R. A. B.).

\section{REFERENCES}

Anderson, K. F., Lonsway, D. R., Rasheed, J. K., Biddle, J., Jensen, B., McDougal, L. K., Carey, R. B., Thompson, A., Stocker, S. \& other authors (2007). Evaluation of methods to identify the Klebsiella pneumoniae carbapenemase in Enterobacteriaceae. J Clin Microbiol 45, $2723-2725$.

CLSI (2008). Performance Standards for Antimicrobial Susceptibility Testing, 18th Informational Supplement. CLSI standard M100-S18. Wayne, PA: Clinical and Laboratory Standards Institute.

Coudron, P. E. (2005). Inhibitor-based methods for detection of plasmid-mediated AmpC $\beta$-lactamases in Klebsiella spp., Escherichia coli, and Proteus mirabilis. J Clin Microbiol 43, 4163-4167.

Jacoby, G. A. \& Munoz-Price, L. S. (2005). The new $\beta$-lactamases. $N$ Engl J Med 352, 380-391.

Jacoby, G. A., Walsh, K. E. \& Walker, V. J. (2006). Identification of extended-spectrum, AmpC, and carbapenem-hydrolyzing $\beta$-lactamases in Escherichia coli and Klebsiella pneumoniae by disk tests. J Clin Microbiol 44, 1971-1976.

Livermore, D. M., Warner, M. \& Mushtaq, S. (2007). Evaluation of the chromogenic Cica- $\beta$-Test for detecting extended-spectrum, AmpC and metallo- $\beta$-lactamases. J Antimicrob Chemother 60, 1375-1379.

Pasteran, F. G., Otaegui, L., Guerriero, L., Radice, G., Maggiora, R., Rapoport, M., Faccone, D., Di Martino, A. \& Galas, M. (2008). Klebsiella pneumoniae carbapenemase-2, Buenos Aires, Argentina. Emerg Infect Dis 14, 1178-1180.

Paterson, D. L., Rice, L. B. \& Bonomo, R. A. (2001). Rapid method of extraction and analysis of extended-spectrum $\beta$-lactamases from clinical strains of Klebsiella pneumoniae. Clin Microbiol Infect 7, 709-711. 
Paterson, D. L., Hujer, K. M., Hujer, A. M., Yeiser, B., Bonomo, M. D., Rice, L. B. \& Bonomo, R. A. (2003). Extended-spectrum $\beta$-lactamases in Klebsiella pneumoniae bloodstream isolates from seven countries: dominance and widespread prevalence of SHV- and CTX-M-type $\beta$ lactamases. Antimicrob Agents Chemother 47, 3554-3560.

Pérez-Pérez, F. J. \& Hanson, N. D. (2002). Detection of plasmidmediated AmpC $\beta$-lactamase genes in clinical isolates by using multiplex PCR. J Clin Microbiol 40, 2153-2162.

Smith Moland, E., Hanson, N. D., Herrera, V. L., Black, J. A., Lockhart, T. J., Hossain, A., Johnson, J. A., Goering, R. V. \& Thomson, K. S. (2003). Plasmid-mediated, carbapenem-hydrolysing $\beta$-lactamase, KPC-2, in Klebsiella pneumoniae isolates. J Antimicrob Chemother 51, 711-714.

Steward, C. D., Rasheed, J. K., Hubert, S. K., Biddle, J. W., Raney, P. M., Anderson, G. J., Williams, P. P., Brittain, K. L., Oliver, A. \& other authors (2001). Characterization of clinical isolates of Klebsiella pneumoniae from 19 laboratories using the National Committee for Clinical Laboratory Standards extended-spectrum $\beta$-lactamase detection methods. J Clin Microbiol 39, 2864-2872.

Tenover, F. C., Raney, P. M., Williams, P. P., Rasheed, J. K., Biddle, J. W., Oliver, A., Fridkin, S. K., Jevitt, L. \& McGowan, J. E., Jr (2003). Evaluation of the NCCLS extended-spectrum $\beta$-lactamase confirma- tion methods for Escherichia coli with isolates collected during Project ICARE. J Clin Microbiol 41, 3142-3146.

Tenover, F. C., Kalsi, R. K., Williams, P. P., Carey, R. B., Stocker, S., Lonsway, D., Rasheed, J. K., Biddle, J. W., McGowan, J. E., Jr \& Hanna, B. (2006). Carbapenem resistance in Klebsiella pneumoniae not detected by automated susceptibility testing. Emerg Infect Dis 12, 1209-1213.

Thomson, K. S., Cornish, N. E., Hong, S. G., Hemrick, K., Herdt, C. \& Moland, E. S. (2007). Comparison of Phoenix and VITEK 2 extendedspectrum- $\beta$-lactamase detection tests for analysis of Escherichia coli and Klebsiella isolates with well-characterized $\beta$-lactamases. J Clin Microbiol 45, 2380-2384.

Wiegand, I., Geiss, H. K., Mack, D., Sturenburg, E. \& Seifert, H. (2007). Detection of extended-spectrum $\beta$-lactamases among Enterobacteriaceae by use of semiautomated microbiology systems and manual detection procedures. J Clin Microbiol 45, 11671174.

Yagi, T., Wachino, J., Kurokawa, H., Suzuki, S., Yamane, K., Doi, Y., Shibata, N., Kato, H., Shibayama, K. \& Arakawa, Y. (2005). Practical methods using boronic acid compounds for identification of class $\mathrm{C}$ $\beta$-lactamase-producing Klebsiella pneumoniae and Escherichia coli. J Clin Microbiol 43, 2551-2558. 